\title{
Extending the Applicability Domain of the Human Cell Line Activation Test (h-CLAT)
}

\author{
Karsten R. Mewes, Ursula Engels, Birgit Eicker and Dirk Petersohn \\ Henkel AG \& Co. KGaA, Düsseldorf, Germany
}

\begin{abstract}
Cosmetic ingredients must be toxicologically assessed to determine their skin sensitizing potential. The in vitro human cell line activation test (h-CLAT; OECD TG 442E) addresses the activation of dermal dendritic cells by analyzing specific protein expression after exposure of THP- 1 cells to the test chemical. According to the protocol, FITC-labeled antibodies are used for protein detection. However, some chemicals show strong autofluorescence at FITC-specific wavelengths so that antibody-specific signals cannot be distinguished appropriately from autofluorescence background. This leads to inconclusive or false-negative predictions. Alternative fluorochromes can be used if their equivalence with the FITC-labeled antibodies is proven. In the current paper we describe the results of a proficiency exercise, based on the proficiency chemicals listed in the guideline, with FITC-labeled antibodies as the benchmark and APC-labeled antibodies as an alternative detection system. APC emits fluorescence at longer wavelengths, thus avoiding interference in the FITC spectrum. Irrespective of the employed fluorochrome, all chemicals were classified correctly, and the EC 150 and 200 values were in the same order of magnitude. Hence, the equivalence in performance of FITC- and APClabeled antibodies was demonstrated, and the respective demand of the guideline was fulfilled. In a case study, we then tested a proprietary oxidative hair dye using both fluorochromes. Using APC-labeled antibodies, the hair dye was unambiguously identified as a sensitizer, whereas no classification could be made with the FITC-labeled antibodies. With APC, fluorescence interference can be circumvented and the applicability domain of the h-CLAT extended to include autofluorescent chemicals.
\end{abstract}

\section{Introduction}

Allergic contact dermatitis (ACD) is a widespread adverse health condition throughout the human population. 8 to $30 \%$ of Europeans develop an ACD during their lifetime depending, e.g., on their country of origin, ethnicity and on the allergens to which they are exposed. Females are far more commonly affected than males (e.g., Peiser et al., 2012; Langen et al., 2013; Diepgen et al., 2016). ACD is responsible for $10-15 \%$ of occupational diseases (Peiser et al., 2012).

In order to protect people from exposure to a potentially sensitizing agent, all ingredients of cosmetics must be toxicologically assessed with respect to their skin-sensitizing potential. To be in line with the European Cosmetics Directive 1223/2009/EU (EU, 2009), the toxicological assessment of chemicals must be conducted by completely animal-free methodologies, relying solely on in vitro, in chemico and in silico approaches.
The physiological mechanisms leading to allergic contact dermatitis (ACD) after exposure to an allergen are described in the adverse outcome pathway (AOP) for skin sensitization (OECD, 2014, 2018). The AOP is characterized by a series of four key events (KE), in which cells of the skin and the immune system are the major players. Upon covalent binding of electrophilic chemicals to cysteine and lysine residues of skin proteins (KE1), keratinocytes are activated, leading to inflammatory reactions and changes in gene expression (KE2). In parallel, Langerhans cells within the skin become activated and migrate out of the epidermis to the next adjacent draining lymph node (KE3). KE4 comprises $\mathrm{T}$ cell activation and proliferation in the lymph node.

Many in vitro test methods addressing one or more key events of the AOP have been developed to date. However, only a few methods have been successfully validated and are now included in OECD testing guidelines (TG), the mandatory gold standard for the mutual acceptance of data among OECD member states.
Received January 24, 2020; Accepted July 31, 2020; Epub August 4, 2020; (C) The Authors, 2020.

ALTEX 38(1), 095-110. doi:10.14573/altex.2001242

Correspondence: Karsten R. Mewes, PhD

Henkel AG \& Co. KGaA

Henkelstr. 67, 40589 Düsseldorf, Germany

(karsten.mewes@henkel.com)
This is an Open Access article distributed under the terms of the Creative Commons Attribution 4.0 International license (http://creativecommons.org/licenses/by/4.0/) which permits unrestricted use, distribution and reproduction in any medium, provided the original work is appropriately cited. 
One such regulatory accepted test method is the human cell-line activation test (h-CLAT), described in OECD TG 442E (OECD, 2018). This method addresses KE3 of the AOP, the activation of dermal dendritic cells (DC) after contact with a sensitizing agent. Briefly, a change in the expression of CD54 and CD86, two cell surface proteins known to be involved in the activation of monocytes and DCs by sensitizers, is analyzed in the human monocytic leukemia cell line THP-1 (Kosaka et al., 2008).

The expression of CD54 and CD86 in THP-1 cells upon exposure to test chemicals is quantified with specific monoclonal antibodies labeled with a fluorophore, which, upon laser excitation in a flow cytometer, emits fluorescent light of a defined wavelength. The original protocol was developed using FITC-labeled antibodies (DB-ALM Protocol No. 158, 2014), and this experimental set-up was adopted in OECD TG 442E.

This assay works well for a multitude of chemicals with different physical-chemical properties (Sakaguchi et al., 2010; Nukada et al., 2012; Takenouchi et al., 2013; EC EURL ECVAM, 2012). However, test items which exhibit strong autofluorescence interfere with the h-CLAT assay. If the wavelength spectrum of the autofluorescence overlaps partially or fully with that of the FITC fluorescence spectrum, one cannot distinguish between the antibody-specific signal and the unspecific background. Thus, the resulting data cannot be interpreted properly, and the prediction must be considered inconclusive. This type of fluorescence interference occurs predominantly with colored chemicals like oxidative hair dyes, which, due to their reactive nature, are potential sensitizers. Although the developers of the h-CLAT gave advice on how to deal with oxidative hair dyes when using the standard h-CLAT protocol, it has become evident that the standard protocol cannot be applied to every autofluorescent test item (Okamoto et al., 2010).

For those cases in which FITC is not compatible with the test item, the guideline allows the use of antibodies with alternative fluorochromes. By choosing fluorochromes with an emission spectrum that clearly differs from the spectral properties of the test item, fluorescence interference can be circumvented for the benefit of clear, specific signals. However, before running the h-CLAT with antibodies coupled to alternative fluorochromes, their equivalence with FITC-labeled antibodies must be unequivocally demonstrated.

The situation can be even more complicated. If the fluorescence of propidium iodide (PI), the dye used to analyze THP-1 cytotoxicity evoked by the test item, also interferes with either the fluorescence spectrum of the test item and/or that of the alternative fluorochrome, the h-CLAT also fails. Alternative cytotoxicity markers are also allowed by the OECD TG as long as they are shown to give similar results to PI.

The equivalence of antibodies with alternative fluorochromes and of alternative cytotoxicity markers with the fluorochromes and dyes of the original protocol must be demonstrated in a proficiency exercise. For this purpose, ten proficiency chemicals are listed in Annex II of the OECD TG 442E. They comprise four non-sensitizers and six sensitizing chemicals covering all potency classes and including liquids and solids. For all proficiency chemicals, reliable in vivo data are available as well as highquality h-CLAT results gained with the original fluorochromes FITC and PI, respectively.

The current paper describes the results of a proficiency exercise according to OECD TG 442E conducted with antibodies against CD54 and CD86 carrying the alternative fluorochrome APC. Based on statistical analyses, we demonstrate the equivalence of antibodies carrying either the FITC or APC fluorochrome with respect to distinguishing between sensitizers and non-sensitizers. This result now allows analyzing the sensitizing potential of autofluorescent molecules using the h-CLAT assay.

The results of a proficiency exercise with alternative cytotoxicity markers, compared to PI, will be published separately.

\section{Material and methods}

All working steps related to the human cell line activation test (h-CLAT) were conducted according to the "h-CLAT DataBase service on Alternative Methods to animal experimentation (DBALM) protocol no. 158" (DB-ALM, 2014), which is the basis for OECD TG 442E (OECD, 2018). The main steps are described briefly below.

\section{Flow cytometer}

Flow cytometry analysis was performed with an Accuri C6 (BD Biosciences, Germany) equipped with two lasers for excitation at $488 \mathrm{~nm}$ and $640 \mathrm{~nm}$. Fluorescence emission was measured with four different optical filters: $530 / 30 \mathrm{~nm}$ (FL1), 585/40 nm (FL2), 670 LP (FL3), and 675/25 nm (FL4). The flow cytometry buffer (MACS-Buffer, Miltenyi, Germany) contained BSA, EDTA and $\mathrm{NaN}_{3}(0.09 \%)$.

Tab. 1: Antibodies used in the study

\begin{tabular}{|l|l|l|}
\hline Isotype control / antibody & Source & Code / Cat. No. \\
\hline Mouse IgG1/FITC, Clone DAK-GO1 & DAKO & X0927 \\
\hline FITC-Mouse Anti-Human CD54; ICAM-1, Clone 6.5B5 & DAKO Code & F7143 \\
\hline FITC-Mouse Anti-Human CD86; Clone 2331 (FUN-1) & Becton Dickinson & 555657 \\
\hline APC-Mouse IgG1 Isotype control; Clone MOPC 21 & Becton Dickinson & 555751 \\
\hline APC-Mouse Anti-Human CD54; Clone HA58 & Becton Dickinson & 559771 \\
\hline APC-Mouse Anti-Human CD86; Clone 2331 (FUN-1) & Becton Dickinson & 555660 \\
\hline
\end{tabular}


Tab. 2: Proficiency chemicals used in the study

\begin{tabular}{|l|l|l|l|}
\hline Proficiency chemical & CAS No. & Source & Order number \\
\hline 2,4-Dinitrochlorobenzene & $97-00-7$ & Aldrich & $23,732-950 \mathrm{~g}$ \\
\hline 1,4-Phenylenediamine & $106-50-3$ & Artec Chemical Co., Ltd. & --- \\
\hline Nickel(II) chloride & $7718-54-9$ & Aldrich & $339350-50 \mathrm{G}$ \\
\hline 2-Mercaptobenzothiazole & $149-30-4$ & Aldrich & M3302-5G \\
\hline R(+)-limonene & $5989-27-5$ & Sigma-Aldrich & $62118-5 \mathrm{ML}$ \\
\hline Imidazoidinyl urea & $39236-46-9$ & Aldrich & I5133-25G \\
\hline Isopropanol & $67-63-0$ & Sigma-Aldrich & $59300-2,5 \mathrm{~L}$ \\
\hline Glycerol & $56-81-5$ & Sigma & G5516-100ML \\
\hline Lactic acid & $50-21-5$ & Sigma & L6661-100ML \\
\hline 4-Aminobenzoic acid & $150-13-0$ & Sigma & A9878-5G \\
\hline
\end{tabular}

\section{Cells and reagents}

The THP-1 cell line, an immortalized human monocytic leukemia cell line, was purchased from the ATCC cell bank, USA. Cells were cultured in RPMI-1640 medium supplemented with $10 \%$ fetal bovine serum, $0.05 \mathrm{mM} 2$-mercaptoethanol, and penicillin and streptomycin. The antibodies used in this study are listed in Table 1. The proficiency chemicals were purchased as indicated in Table 2.

\section{Cell culture and reactivity check}

Performance of the cultured THP-1 cells was assessed in a reactivity check. The cells were exposed for 24 hours to 2,4-dinitrochlorobenzene $(2,4-\mathrm{DNCB})$ or nickel sulfate $\left(\mathrm{NiSO}_{4}\right)$ as positive controls and to lactic acid as a negative control. Whereas 2,4-DNCB and $\mathrm{NiSO}_{4}$ should induce marked up-regulation of CD54 and CD86 expression, no induction should occur with lactic acid. In addition, cell viability of untreated THP-1 cells should not drop below $90 \%$. Only when the acceptance criteria were met, the cells were qualified to be used in the h-CLAT assay. The reactivity check was conducted for every freshly thawed THP-1 cell batch employed in this study. THP-1 cells, cultured in passages 6 to 23, were employed for dose-range finding assays and chemical testing.

\section{Dose-finding assay (PI assay)}

Serial dilutions for each test chemical were established either in saline, starting with a maximum concentration of $5000 \mu \mathrm{g} / \mathrm{mL}$, or in DMSO, starting with a maximum concentration of $1000 \mu \mathrm{g} /$ $\mathrm{mL}$. THP-1 cells were incubated for 24 hours with each solution, and cell viability was determined with PI. For more information about the appropriate flow cytometer set-up, please refer to the DB-ALM protocol no. 158. Cell viability was calculated according to the following formula (Eq. 1):

Cell viability $=\frac{\text { Number of living cells }}{\text { Total number of acquired cells }} \times 100$
Based on the cell viability data, the CV75, i.e., the chemical concentration at which $75 \%$ cell viability is achieved, was calculated by log-linear interpolation according to the following formula (Eq. 2):

$\log C V 75=\frac{(75-c) \times \log (b)-(75-a) \times \log (d)}{a-c}$ Eq. 2

The CV75 sets the starting point for preparing the serial dilutions of the chemical in the h-CLAT assay, always defining the second-highest concentration in the dilution series. In the case that no cytotoxicity is observed even at the highest concentration, the serial dilutions (stock solutions) start with $5000 \mu \mathrm{g} / \mathrm{mL}$ in saline or with $1000 \mu \mathrm{g} / \mathrm{mL}$ in DMSO, respectively, as the maximum concentrations, depending on chemical solubility.

\section{Chemical testing}

Briefly, the stock saline-based solutions were diluted 1:50 and the DMSO-based solutions 1:250 in culture medium (working solutions). THP-1 cells, suspended in fresh cell culture medium, were added to the wells of a 24 -well flat-bottom plate with a final number of $1 \times 10^{6}$ cells per well. Then $500 \mu \mathrm{L}$ of each working solution was pipetted into the cell suspension, resulting in the final chemical concentration. The cells were then cultured for 24 hours at $37^{\circ} \mathrm{C}$ and $5 \% \mathrm{CO}_{2}$ in an incubator. In addition, a medium and DMSO control were included as well as $2,4-\mathrm{DNCB}$ as a positive control.

After 24 hours, cells were collected by centrifugation, washed twice with $1 \mathrm{~mL}$ FACS buffer, and blocked with $500 \mu \mathrm{L}$ blocking solution. Then the cells were divided into 3 aliquots of $180 \mu \mathrm{L}$ each in a 96-well plate and centrifuged. After discarding the supernatant, $50 \mu \mathrm{L}$ pre-mixed staining solution containing the respective antibodies ( $6 \mu \mathrm{L}$ anti-CD86 antibody or $3 \mu \mathrm{L}$ anti-CD54 antibody or $3 \mu \mathrm{L}$ isotype control) were added to each cell pellet. The cells were incubated with the antibody solutions for $30 \mathrm{~min}$ at $4{ }^{\circ} \mathrm{C}$ in the dark and washed twice with flow buffer. For cell 
viability assessment, cells were resuspended in $200 \mu \mathrm{L}$ flow buffer containing $0.625 \mu \mathrm{g} / \mathrm{mL}$ PI.

Expression of the cell surface antigens CD54 and CD86 and cytotoxicity were analyzed by flow cytometry, strictly following the DB-ALM protocol, but also taking the special requirements of the used flow cytometer into account. The measure for CD54 or CD86 expression was the mean fluorescence intensity (MFI), for cytotoxicity it was the percentage of dead cells.

As an indicator for CD54 and CD86 expression, the relative fluorescence intensity (RFI) was calculated on the basis of the MFI values according to the following equation (Eq. 3):

$$
\begin{aligned}
& \text { (MFI of chemical treated cells - } \\
& \mathrm{RFI}=\frac{\text { MFI of chemical treated isotype cells) }}{\text { MFI of solvent treated isotype cells) }}
\end{aligned}
$$

To be qualified and thus included for further analyses, the following acceptance criteria had to be fulfilled:

- Cell viability of medium and DMSO control should be more than $90 \%$.

- RFI values of the positive control for both CD54 and CD86 should be above the respective positive criteria CD54 $\geq 200$ and CD86 $\geq 150$, and cell viability should be higher than $50 \%$.

- RFI values of the DMSO solvent control should not exceed the positive criteria.

- For both medium and DMSO control, the mean fluorescence intensity (MFI) ratio of CD to isotype control should be $>105 \%$.

All runs conducted in the present study fulfilled the quality criteria.

\section{Calculation of EC150 and EC200}

The concentrations at which a sensitizing test chemical induces a $150 \%$ or $200 \%$ expression of CD54 and CD 86 , respectively, i.e., the EC150 and EC200 values, can be calculated either by linear interpolation or by log-linear extrapolation, depending on the position of the putative EC values in relationship to the dilutions tested. All equations needed for calculation and the criteria for when to use which mathematical approach are described in the DB-ALM protocol and are not repeated here due to complexity.

\section{The h-CLAT prediction model}

Every chemical was tested in at least 2 independent runs that fulfilled all quality criteria. According to DB-ALM protocol no. 158, the prediction model is defined as follows: "If the RFI of CD86 is equal to or greater than $150 \%$ at any tested dose $>50 \%$ of cell viability) in at least 2 independent runs, AND/OR if the RFI of CD54 is equal to or greater than 200\% at any tested dose (> 50\% of cell viability) in at least 2 independent runs, the chemical prediction is considered as positive. Otherwise it is considered as a negative. In case the first two independent runs are not concordant, a third run needs to be performed, and the final prediction will be based on the majority of the results from three individual runs (i.e., 2 out of 3 )."

\section{Fluorescence check}

THP-1 cells were exposed to the test chemical at 8 different dilutions, with CV75 as starting point, for 24 hours. After 24 hours, the cells were collected by centrifugation, washed twice with $1 \mathrm{~mL}$ FACS buffer, centrifuged again and resuspended in $200 \mu \mathrm{L}$ FACS buffer. Then the cells were subjected to flow cytometry analysis. Fluorescence was determined with all laser/detection channel combinations available in the respective flow cytometer. In the BD Accuri, 4 different combinations, FL1-A to FL4-A, could be used. The relative fluorescence intensities gained in all laser/ channel combinations were then plotted against the chemical concentrations. The laser/channel combination with the lowest fluorescence signals over the whole concentration range was selected for further analysis.

\section{Statistics}

As the data were not normally distributed, the correlation analysis between the FITC- and APC-generated data sets was conducted by applying Spearman's rank correlation method. A correlation was considered significant when Spearman's coefficient $\rho$ was $>0.3$ or $<-0.3$.

\section{Results}

\subsection{Comparison of FITC versus APC fluorophores for CD86 and CD54}

\subsubsection{Validity of flow cytometry analysis}

Every proficiency chemical listed in Appendix II of OECD TG 442E was tested with FITC-labeled and APC-labeled antibodies against CD54 and CD86 in the same test set. In addition, non-treated THP-1 cells as negative control (NC) and cells exposed to $\mathrm{DNCB}$ as positive control (PC) were analyzed in each run, as indicated in OECD TG 442E. Representative histograms of the NC and $\mathrm{PC}$ for CD54, CD86 and the isotype control are shown in Figure 1.

In the NCs, the signals for CD54, CD86 and the isotype controls were nearly indistinguishable in both the FL1 channel (FITC detection) and the FL4 channel (APC detection); no specific marker expression was observed (Fig. 1a,b). On average, the MFI for CD86 was higher than the MFI for CD54 with the FITC-labeled antibodies, whereas CD54 was higher than CD86 with APC. In general, FITC revealed higher fluorescence emission levels than APC independent of antibody specificity (Fig. 1e). The PC markedly induced CD54 and CD86 (Fig. 1c,d). Thus, the mandatory quality criteria were fulfilled, and the respective test runs qualified. This was the case for all h-CLAT test runs documented in this study. Without antibody staining, the THP-1 cells revealed a certain degree of autofluorescence, with higher MFI values in the FITC-specific FL1 channel compared to rather low values in the FL4 channel used for APC detection (Fig. 1f).

\subsubsection{Correlation analysis of MFI values}

The MFI values for all tested chemical concentrations and for all valid test runs were included in the statistical analysis to determine how closely the FITC- and the APC-based values cor- 

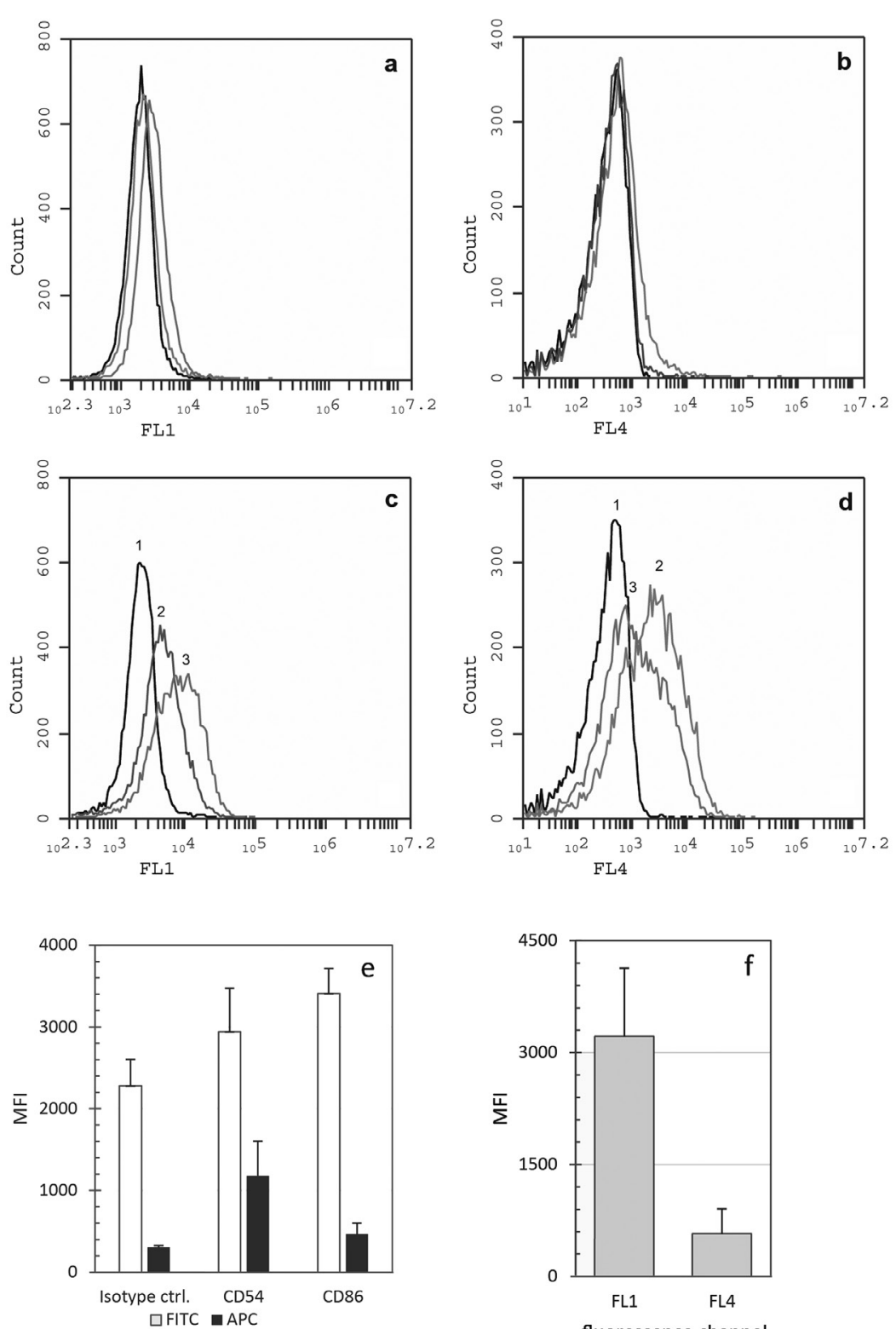

Fig. 1: Fluorescence properties of untreated cells and positive controls (a-d) Representative histograms of the mean fluorescence intensities for untreated (NC; a,b) or DNCB-treated (PC; c,d) THP-1 cells incubated with FITC-labeled (a,c) or APC-labeled (b,d) antibodies against CD54 and CD86 and for the respective isotype controls. The histograms in (c) and (d) are labeled as indicated: 1) isotype control; 2) CD54; 3) CD86. (e) MFI of untreated cells stained with FITC- or APClabeled anti-CD54, anti-CD86 or isotype control antibodies ( $n=5$, mean + SD). (f) Autofluorescence of untreated, unlabeled THP-1 cells in the flow cytometry channels FL1 and FL4, expressed as mean fluorescence intensity $(\mathrm{MFl} ; \mathrm{n}=5$, mean $+\mathrm{SD})$. relate. With this analysis the specific performance, or fluorescence emission, of the antibodies in flow cytometry, based solely on their different fluorochromes, was assessed. We used Spearman's rank correlation method because the data, due to their nature, do not follow the Gaussian distribution.

In a first analysis, we compared the MFI values irrespective of the sensitizing potential of the tested chemicals. The data sets generated with FITC and APC for CD54 and CD86 were significantly correlated $(\mathrm{p}<0.05)$, with a slightly higher correlation coefficient for the CD86 values (Tab. 3). The data points were unevenly distributed in the x-y correlation plots (Fig. 2a,d). An aggregation of data, representing the majority of non-sensitizers, is observed in the lower left corner, whereas a second group of data, mainly consisting of the sensitizers, are evenly aligned along the linear correlation slope.

In a next step, we analyzed the values generated for the non-sensitizers and sensitizers separately. As indicated by a higher Spearman's correlation coefficient, the correlation between FITC- and APC-labeled antibodies was stronger for sensitizers than for non-sensitizers for both CD54 and CD86 (Tab. 3). The grouping of values according to the sensitization potential of the chemicals is visualized in more detail in $\mathrm{x}-\mathrm{y}$ plots (Fig. 2). The low MFI values for the non-sensitizing chemicals are confined within a narrow range. In contrast, the MFI data representing the sensitizing substances are distributed over a much wider range of values according to the widely varying induction levels for sensitizers of differ- 

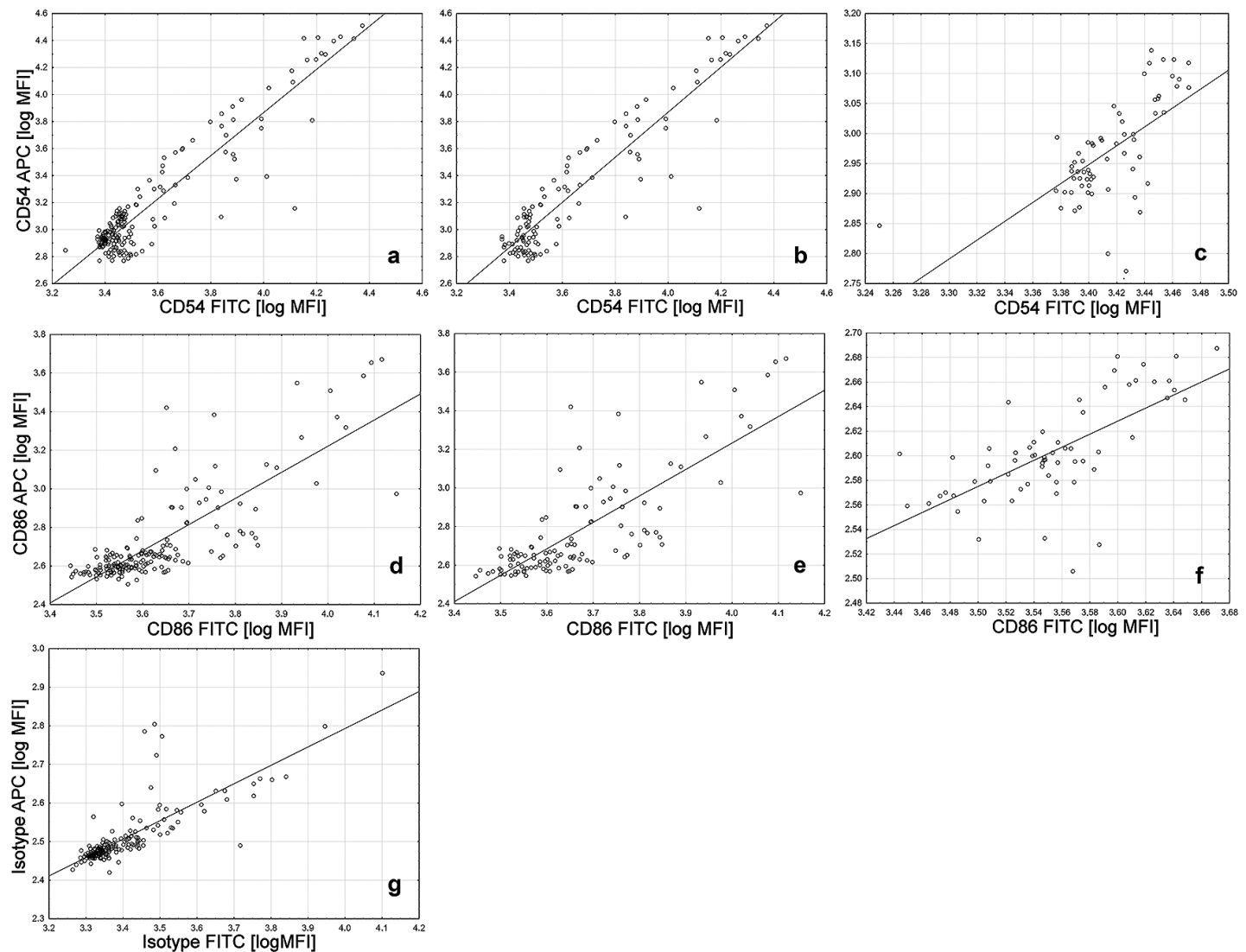

Fig. 2: Correlations between mean fluorescence intensity (MFI) values generated with either FITC-labeled (x-axis) or APC-labeled antibodies (y-axis) against CD54 (a-c) or CD86 (d-f)

MFI values are plotted in a double-logarithmic scale to include the wide range of values. MFI values for all chemicals tested (a,d); MFI values for all sensitizers (b,e); MFI values for all non-sensitizers (c,f). Correlation between the FITC- and APC-labeled isotype controls $(\mathrm{g})$. The linear correlation line is shown for each data set.

ent potencies. Whereas an overlap between the absolute MFI values for sensitizers and non-sensitizers is apparent in the group of low values, the higher MFI values are exclusively attributed to the sensitizing chemicals. The MFI data of the isotype controls, measured in parallel to each run with either FITC- or APC-labeled antibodies, are strongly correlated with each other (Tab. 3; Fig. 2g), with generally higher values observed in the FL1 channel for FITC detection than in the FL4 channel used for APC detection.

In spite of the high correlations between the results generated with FITC- and APC-labeled antibodies, the numerical values of the MFI data were not identical. On average, the MFI values for the FITC-based analyses were higher than the APC data. To analyze this observation in more detail, the MFI values for APC were subtracted from the corresponding FITC values, and the resulting differences were plotted in $\mathrm{x}-\mathrm{y}$ diagrams for CD54 and CD86, respectively (Fig. 3). For CD54, the vast majority of values were positive within a fairly narrow range of values of 1000 and 3000 for both sensitizers and non-sensitizers (Fig. 3a-c). Higher val-
Tab. 3: Correlation coefficients for pairwise comparisons of MFI values for FITC- and APC-labelled antibodies against CD54 and CD86

In addition, the correlation coefficient for the isotype controls is listed. For all correlation coefficients: $p<0.05$.

\begin{tabular}{|l|l|l|}
\hline Spearman's correlation coefficient & CD54 & CD86 \\
\hline All chemicals & 0.694 & 0.749 \\
\hline Sensitizers & 0.821 & 0.746 \\
\hline Non-sensitizers & 0.663 & 0.649 \\
\hline Isotype control & \multicolumn{2}{|c|}{0.818} \\
\hline
\end{tabular}

ues were calculated only within the group of sensitizers (Fig. 3b), mainly related to the moderate sensitizer 2-mercaptobenzothiazolol (2-MBT). Some differences were negative, which means higher MFI values for APC-labeled antibodies. These data were 

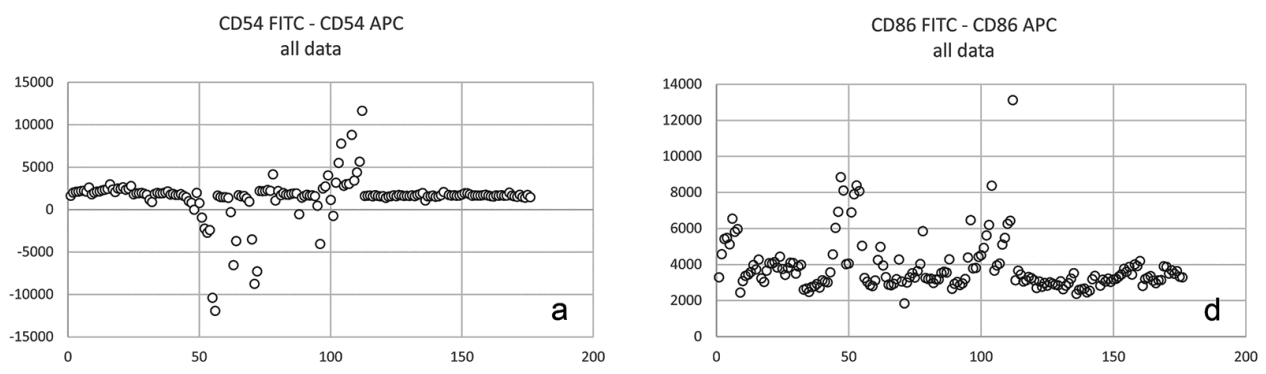

Fig. 3: Differences

of MFI values from

APC-labeled samples subtracted from MFI values from FITC-labeled samples for CD54 (a-c) and CD86 (d-f)

Differences are shown for all chemicals tested $(a, d)$, for the sensitizers $(b, e)$, and for the nonCD54 FITC - CD54 APC Sensitizer
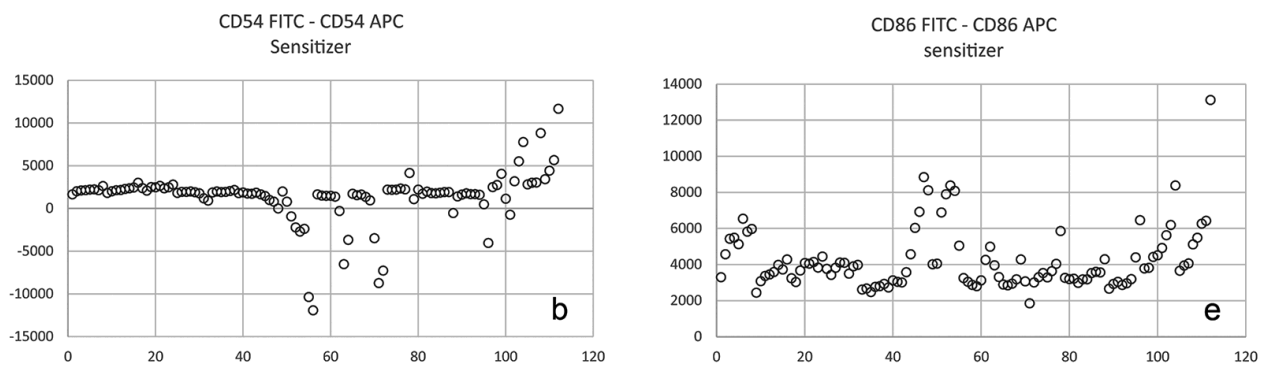

sensitizers (c,f).

$X$-axis, number of chemicals; $y$-axis, MFI units
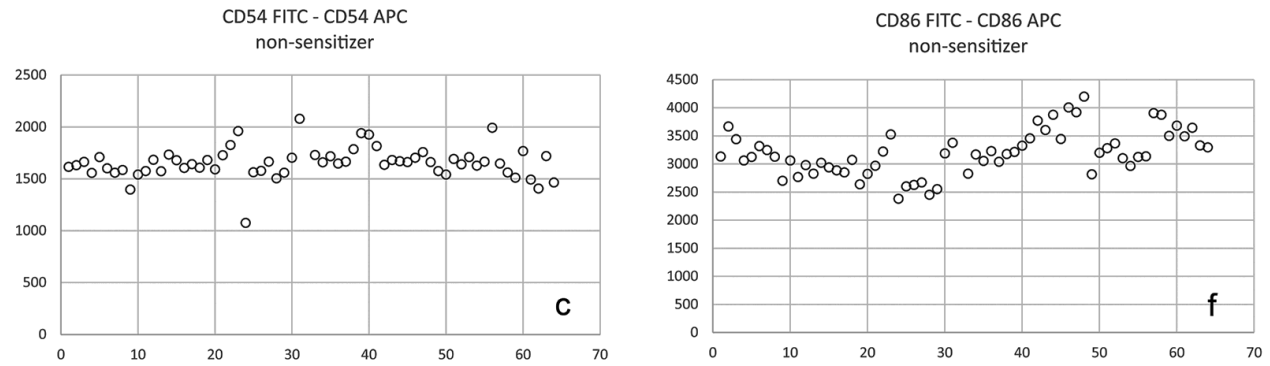

mainly allocated to the moderate sensitizer $\mathrm{NiSO}_{4}$ and to $\mathrm{DNCB}$, an extreme sensitizer.

With CD86, all FITC-APC differences were clearly positive (Fig. 3d-f). Whereas the values for the non-sensitizers were confined to a range between 2000 and 4500 (Fig. 3f), the values were more variable for the sensitizing chemicals. Higher differences above 4500 could be attributed to 2-MBT, DNCB and the strong sensitizer 1,4-phenylenediamine (1,4-PD). For all chemicals tested, the MFI for FITC was higher than the corresponding APC value.

\subsubsection{Concordance of classification}

Correlation analysis of RFI values

The final classification of a chemical as a sensitizer or non-sensitizer is based on the RFI value, which is defined as the difference between the MFI value generated with the FITC and APC-labeled antibodies, respectively, and the MFI value of the corresponding isotype control, both corrected for the respective solvent control.
In order to assess whether the final classification was affected by the type of fluorochrome used in the h-CLAT assay, we analyzed the correlation between the FITC- and APC-based RFI values. Table 4 lists the correlation coefficients for all chemicals, and separately for the sensitizers and non-sensitizers.

The data sets generated with FITC- and APC-labeled antibodies against CD54 and CD86 are significantly correlated ( $<<0.05)$, irrespective of their skin-sensitizing potential, with a slightly higher correlation coefficient for the CD54 data. In the x-y plots, most RFI data points accumulate at fairly low values, visualized as data clouds in the lower left parts of the diagrams (Fig. 4a,d), while a smaller subgroup of data is scattered over a broader range of values, but close to the regression line. The degree of correlation is even higher when focusing only on the data generated with the sensitizing chemicals, with the majority of data points in the low value range (Fig. 4b,e). In contrast, Spearman's correlation coefficients for the non-sensitizers were low for both CD54 and CD86. The RFI data points in the corresponding $x-y$ plots are scattered in 

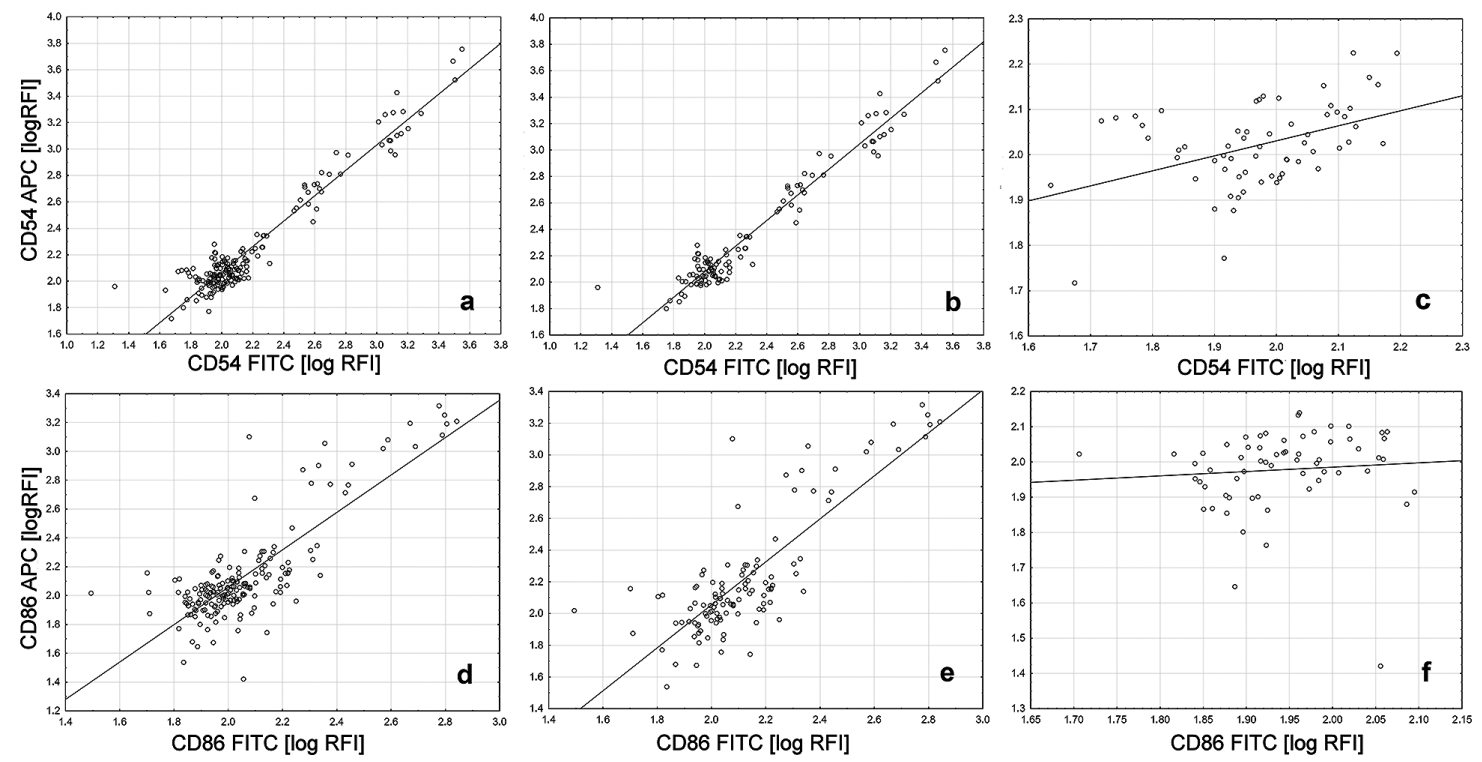

Fig. 4: Correlations between the relative fluorescence intensity (RFI) values generated with either FITC-labeled (x-axis) or APC-labeled (y-axis) antibodies against CD54 (a-c) and CD86 (d-f)

RFI values are plotted in a double-logarithmic scale to include the wide range of values. RFI values for all chemicals tested (a,d); RFI values for all sensitizers (b,e); RFI values for all non-sensitizers (c,f). The linear correlation line is shown for each data set.

an apparently random pattern in the low value ranges (Fig. 4c,f). In the low value ranges, the data for sensitizers and non-sensitizers overlap, whereas the data representing higher RFI values are exclusively attributed to the sensitizers.

\section{Classification}

Every proficiency chemical listed in Appendix II of OECD TG 442E, comprising 4 non-sensitizers and 6 sensitizers of different sensitizing potency, was tested in at least 2 independent runs, which fulfilled all quality criteria for acceptance as valid (DBALM, 2014). We then analyzed how many of the test items had been predicted correctly with either the FITC- or the APC-labeled antibodies and how the results for both fluorophores were correlated. As the h-CLAT is officially approved only for hazard classification, which means to distinguish between being a sensitizer or a non-sensitizer, the respective potency classes of the chemicals were not considered for this analysis. The dose response curves for all chemicals and all runs are presented in Figure $\mathrm{S}^{1}{ }^{1}$. All chemicals were predicted correctly with both FITC and APC-labeled antibodies, resulting in a $100 \%$ correlation (Tab. 5).

\section{Calculation of EC150 and EC200}

The EC200 and EC150 values define the effective chemical concentrations at which CD54 and CD86 are expressed at 200\% and $150 \%$ of the solvent control, respectively. Hence, they can be con-
Tab. 4: Correlation coefficients for pairwise comparisons of the RFI values for FITC- and APC- labelled antibodies against CD54 and CD86

For all comparisons: $p<0.05$.

\begin{tabular}{|l|l|l|}
\hline Spearman's correlation coefficient & CD54 & CD86 \\
\hline All chemicals & 0.779 & 0.638 \\
\hline Sensitizers & 0.858 & 0.704 \\
\hline Non-sensitizers & 0.412 & 0.284 \\
\hline
\end{tabular}

sidered measures for the skin-sensitizing potency of the tested chemicals.

Figure 5 compares the EC200 values of CD54 calculated for the FITC and APC approaches for the sensitizing chemicals. For 5 out of 6 chemicals, the mean EC200 values, based on 2 or 3 test runs, respectively, were in the same range of values, with $\mathrm{R}(+)$-limonene having the highest and 2,4-DNCB the lowest values. Exposure of the THP-1 cells to 1,4-PD did not elicit CD54 induction at any tested concentration; all RFI values were clearly below the induction threshold of 200.

Figure 6 illustrates the EC150 values of CD86 for both FITC and APC approaches for the 6 skin-sensitizing chemicals. The EC150 values for nickel chloride, imidazoidinyl urea and

1 doi:10.14573/altex.2001242s 


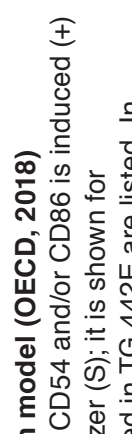

은 논

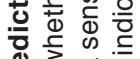

흐웜

ब

은

흥ㅎํㅎํ

응 성

它

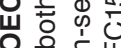

흐음

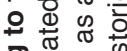

을

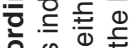

엉

की

ฮั่ 仓ั

충웡

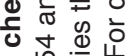

>뉸

वेग क्ष

$\div$ के

흔

중 $\overline{0}$

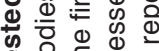

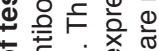

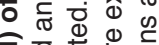

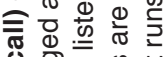

웜

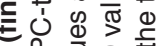

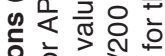

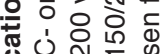

ฮํํ용

证它

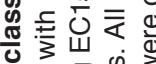

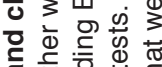

ब声产

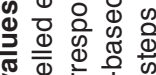

究

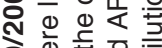

뉵응

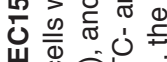

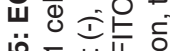

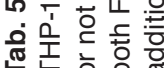

\begin{tabular}{|c|c|c|c|c|c|c|c|c|c|c|c|}
\hline \multicolumn{2}{|l|}{ ّْ̊. } & $\stackrel{\infty}{\nu}$ & $\stackrel{\infty}{\infty}$ & $\stackrel{\infty}{\infty}$ & $\stackrel{\infty}{\stackrel{\infty}{\infty}}$ & $\stackrel{\infty}{\check{\nu}}$ & $\stackrel{\infty}{ల}$ & $\stackrel{\infty}{\nu}$ & $\stackrel{\infty}{\varnothing}$ & $\stackrel{\infty}{\stackrel{\infty}{\infty}}$ & $\stackrel{\infty}{\rightleftharpoons}$ \\
\hline 㞼 $\overline{\bar{~}}$ & $\begin{array}{l}0 \\
\vdots \\
\frac{\alpha}{\alpha} \\
\text { 는 } \\
\text { 는 }\end{array}$ & $\frac{\infty}{\infty}$ & $\frac{\omega}{\omega}$ & $\frac{\infty}{\omega}$ & $\frac{\infty}{\omega}$ & $\frac{\infty}{\omega}$ & $\frac{\infty}{\omega}$ & 面 & $\sum_{\infty}^{\infty}$ & $\underset{\text { NO }}{\stackrel{\infty}{Z}}$ & $\sum_{\text {ND }}^{\infty}$ \\
\hline 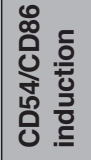 & 选 & $\stackrel{t}{+}$ & \pm & $\stackrel{+}{+}$ & \pm & $\frac{1}{+}$ & $\stackrel{+}{+}$ & $\div$ & $\div$ & $\div$ & $\div$ \\
\hline 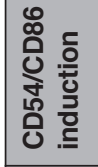 & $\frac{U}{\frac{U}{4}}$ & $\stackrel{+}{+}$ & \pm & $\stackrel{+}{+}$ & $\stackrel{+}{+}$ & $\frac{1}{+}$ & $\stackrel{+}{+}$ & $\div$ & $\frac{1}{1}$ & $\div$ & $\div$ \\
\hline ن & 遂 & $\stackrel{\infty}{\nu}$ & $\stackrel{\infty}{=}$ & $\stackrel{\infty}{=}$ & $\stackrel{\mathscr{\infty}}{\rightleftharpoons}$ & io & $\stackrel{\infty}{>}$ & $\stackrel{\infty}{>}$ & $\stackrel{\mathscr{\infty}}{\rightleftharpoons}$ & 足 & $\stackrel{\mathscr{\infty}}{\rightleftharpoons}$ \\
\hline 党嵌 & 㫐 & $\stackrel{\infty}{\infty}$ & $\stackrel{\infty}{=}$ & $\stackrel{\infty}{>}$ & $\stackrel{\infty}{=}$ & io & $\stackrel{\infty}{>}$ & $\stackrel{\infty}{>}$ & $\stackrel{\infty}{>}$ & $\begin{array}{l}0 \\
2 \\
2\end{array}$ & $\stackrel{\infty}{=}$ \\
\hline 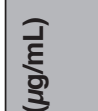 & $\frac{0}{4}$ & $\stackrel{?}{\leftarrow}$ & $\begin{array}{l}\text { fo } \\
\text { ì } \\
\end{array}$ & $\begin{array}{l}\qquad \\
\circ \\
6 \\
6 \\
\end{array}$ & $\begin{array}{l}\infty \\
\dot{f} \\
\dot{f}\end{array}$ & $\dot{c}$ & 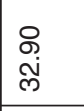 & $\begin{array}{l}8 \\
8 \\
1 \\
1 \\
\end{array}$ & $\begin{array}{l}8 \\
8 \\
10 \\
1\end{array}$ & $\begin{array}{c}\underset{+}{+} \\
\stackrel{+}{f} \\
\Lambda\end{array}$ & $\begin{array}{l}8 \\
\\
1 \\
\end{array}$ \\
\hline $\begin{array}{l}\text { L } \\
\text { L } \\
\end{array}$ & 은 & 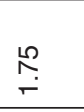 & $\begin{array}{l}0 \\
0 \\
0\end{array}$ & $\begin{array}{l}8 \\
\dot{i} \\
\end{array}$ & $\dot{\check{c}}$ & $\dot{\Sigma}$ & 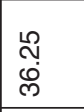 & $\begin{array}{l}8 \\
8 \\
1 \\
1 \\
\end{array}$ & $\begin{array}{l}8 \\
0 \\
10 \\
1 \\
\end{array}$ & 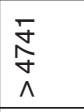 & $\begin{array}{l}8 \\
\\
1 \\
\end{array}$ \\
\hline हु & 遈 & $\stackrel{\substack{\infty \\
+}}{+}$ & 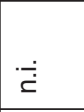 & $\begin{array}{l}1 \\
\infty \\
0 \\
0 \\
\end{array}$ & 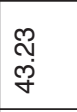 & 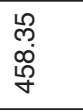 & $\begin{array}{l}0 \\
9 \\
0 \\
e \\
\end{array}$ & $\begin{array}{l}8 \\
80 \\
10 \\
1\end{array}$ & $\begin{array}{l}8 \\
0 \\
10 \\
1\end{array}$ & 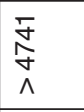 & $\begin{array}{l}8 \\
\\
\\
\end{array}$ \\
\hline 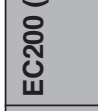 & 은 & $\stackrel{\infty}{\stackrel{\infty}{\Gamma}}$ & ¿ & $\begin{array}{l}8 \\
\infty \\
\infty \\
\end{array}$ & $\begin{array}{l}\hat{o} \\
\infty \\
\infty \\
\end{array}$ & $\begin{array}{l}\stackrel{\infty}{\rho} \\
\stackrel{0}{\circ} \\
\stackrel{\circ}{\circ}\end{array}$ & 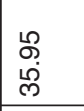 & $\begin{array}{l}8 \\
80 \\
1 \\
1\end{array}$ & \begin{tabular}{|l}
8 \\
0 \\
10 \\
1 \\
\end{tabular} & \begin{tabular}{c}
$\bar{J}$ \\
\multirow{J}{*}{} \\
$\Lambda$
\end{tabular} & $\begin{array}{l}8 \\
\\
1\end{array}$ \\
\hline 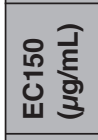 & 岁 & 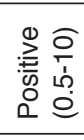 & 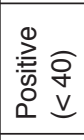 & 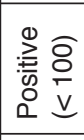 & 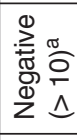 & 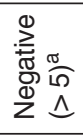 & 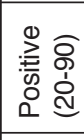 & 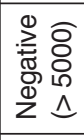 & 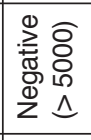 & 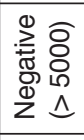 & 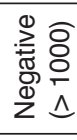 \\
\hline 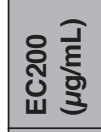 & 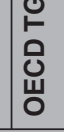 & 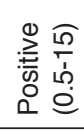 & 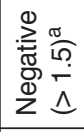 & 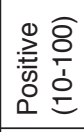 & 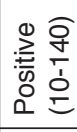 & 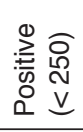 & 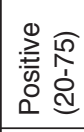 & 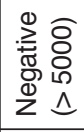 & 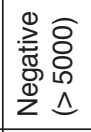 & 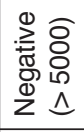 & 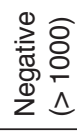 \\
\hline 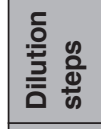 & & 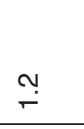 & $\stackrel{O}{\mathrm{i}}$ & $\stackrel{\infty}{\stackrel{\infty}{-}}$ & 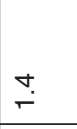 & $\stackrel{\circ}{\mathrm{N}}$ & $\stackrel{m}{\check{r}}$ & $\stackrel{\circ}{-}$ & $\stackrel{\text { L }}{\longrightarrow}$ & 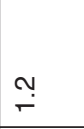 & $\stackrel{m}{-}$ \\
\hline 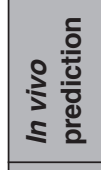 & & 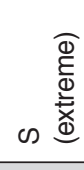 & os & $\begin{array}{l}\frac{\Phi}{\pi} \\
\frac{\pi}{\frac{\pi}{0}} \\
\omega\end{array}$ & 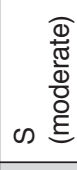 & 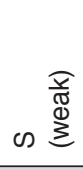 & $\omega \stackrel{\text { }}{\text { I্ }}$ & $\frac{0}{2}$ & $\frac{\infty}{z}$ & $\stackrel{0}{z}$ & $\stackrel{0}{z}$ \\
\hline 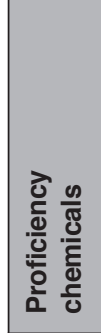 & & 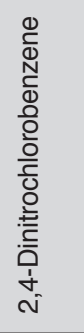 & 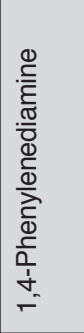 & 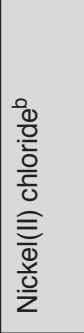 & 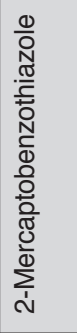 & 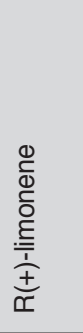 & 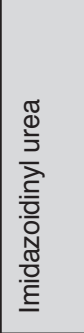 & \begin{tabular}{|l}
$\overline{0}$ \\
$\frac{0}{\pi}$ \\
$\frac{0}{0}$ \\
$\frac{0}{0}$ \\
$\underline{0}$ \\
$\underline{0}$ \\
\end{tabular} & 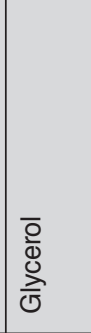 & 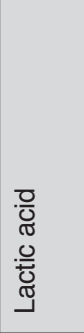 & 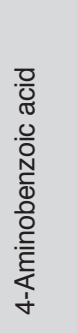 \\
\hline
\end{tabular}

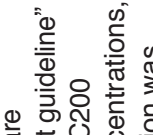

厅

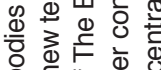

旁 ฮ

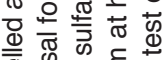

क्ष

نं 흥호 흘

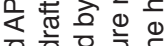

흥하

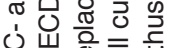

잉

एँ.

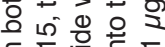

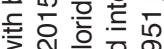

$\subseteq$ 등.

정 층 흥

वृ के $\frac{1}{5}$.

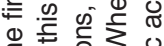

क्ष

ब

응

을

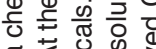

ฮ艹

훙

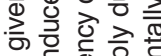

$\underline{\infty}=$.

\&

뜡 @ 는

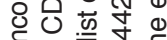

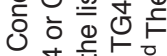

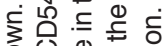

은 0.5 .0

क

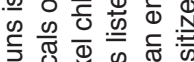

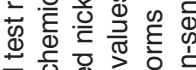

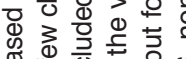

कू

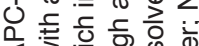

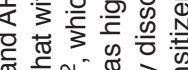

का

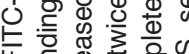

प

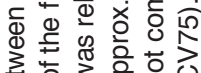

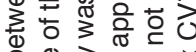

일

U.

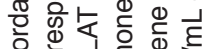

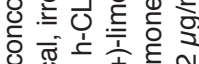

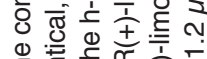

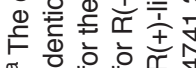

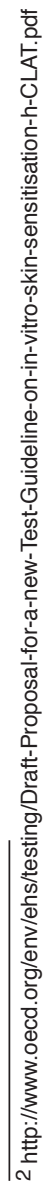



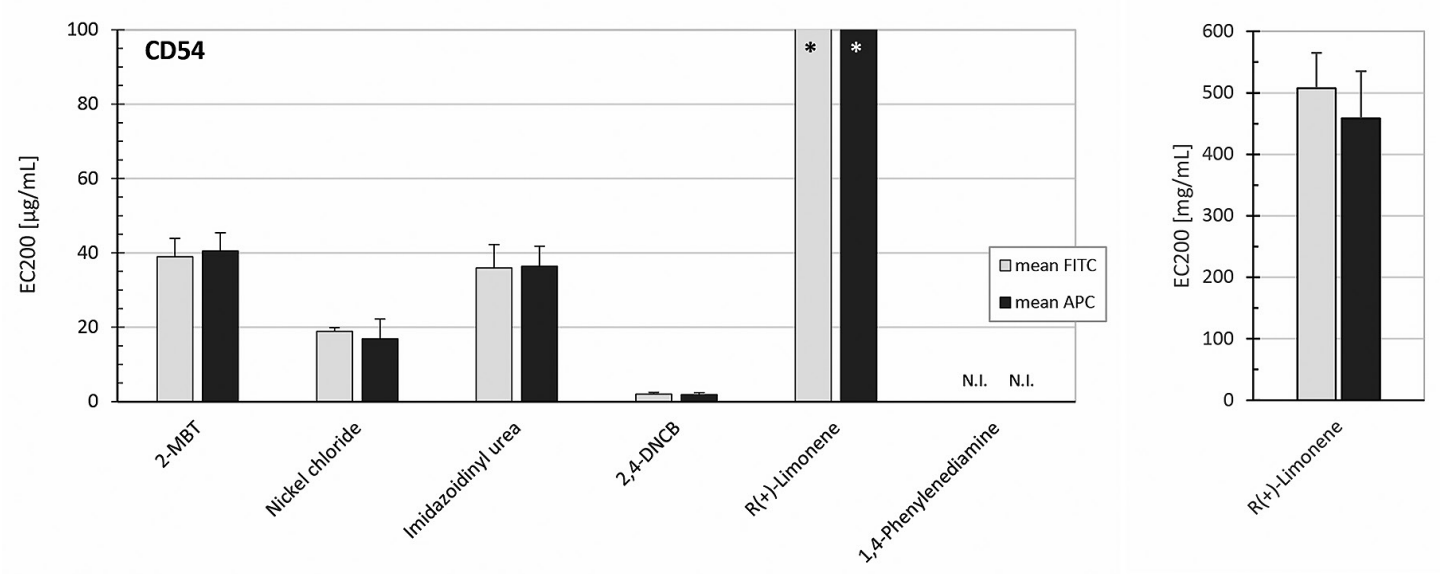

Fig. 5: EC200 values calculated for the 6 sensitizing chemicals

Each bar represents the mean of 2 (3) independent test runs. N.I., no induction measured. *As the induction values for $\mathrm{R}(+)$-limonene exceeded $100 \mu \mathrm{g} / \mathrm{mL}$, which is the upper limit in Fig. 3 and 4 based on the range of values for all other chemicals, they are shown separately as complete bars in the additional diagram.

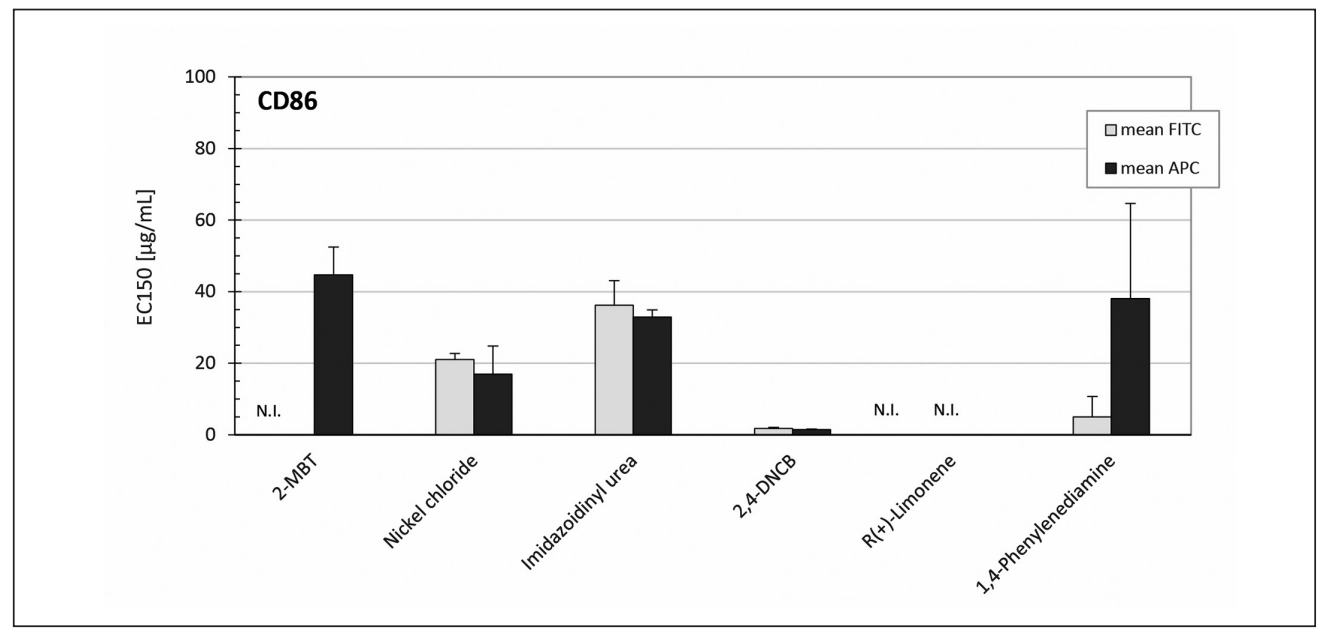

Fig. 6: EC150 values calculated for the 6 sensitizing chemicals Each bar represents the mean of 2 (3) independent test runs. N.I., no induction measured.

2,4-DNCB were all in the same range of values, whereas $\mathrm{R}(+)$-limonene did not induce CD86 in any approach. The EC150 values for 1,4-PD differed markedly between FITC and APC approaches $(5 \mu \mathrm{g} / \mathrm{mL}$ with the FITC-labeled antibodies, $38 \mu \mathrm{g} / \mathrm{mL}$ with APC-tagged antibodies). In order to identify possible reasons for this discrepancy, the RFI values for all 1,4-PD concentrations tested in all 3 runs were analyzed in more detail, as illustrated in Figure 7a. No concentration dependency of CD86 induction was observed, neither with FITC nor with APC. Instead, most values were closely clustered around the RFI induction threshold of 150 .

CD54 induction by 2-MBT was only detected with the APC-labeled antibody. When analyzing the individual RFI values, no clear concentration dependency of CD54 expression was observed, neither for FITC nor for APC (Fig. 7b). Some of the FITC values nearly reached the $150 \%$ threshold, thus no EC 150 calculation was possible. In contrast, with some of the APC values crossing the threshold, an EC150 value could be determined.

The effective chemical concentrations (EC200 and EC150) for CD54 and CD86 induction were in similar ranges for 2-MBT, nickel chloride, imidazoidinyl urea and 2,4-DNCB.

\subsection{Case study: Hair dye testing with FITC- and APC-labeled antibodies}

We clearly demonstrated the equivalence between FITC- and APC-labeled antibodies in assessing the skin-sensitizing potential of chemicals with the proficiency exercise, thus fulfilling the key demand of OECD TG 442E for using alternative fluorochromes. From previous h-CLAT studies we knew that certain classes of oxidative hair dyes cannot be assessed properly with FITC-labeled antibodies. We hypothesized that these specialty chemicals emit autofluorescence, based on their chemical struc- 


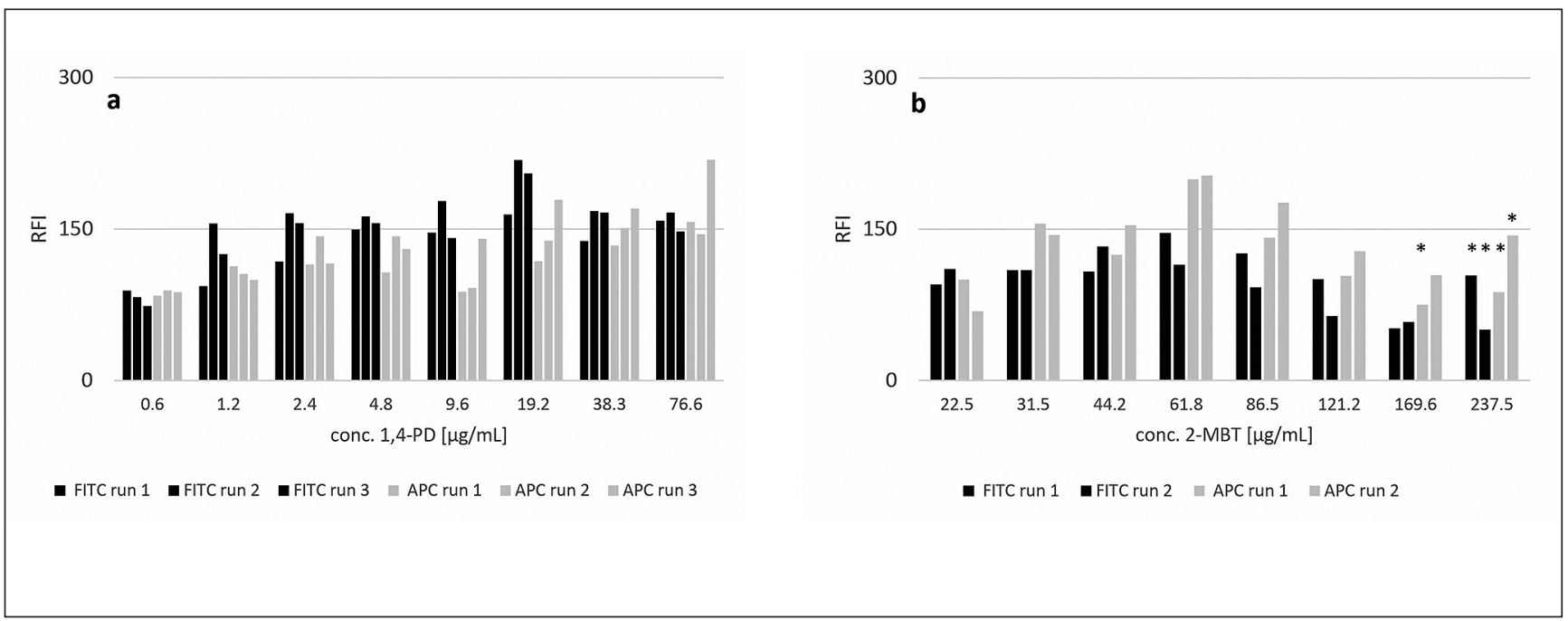

Fig. 7: Compilation of the RFI values for all 1,4-PD (a) and 2-MBT (b) concentrations [in $\mu \mathrm{g} / \mathrm{mL}$ ] tested in 3 (2) independent runs with FITC- or APC-labeled anti-CD86 antibodies

The threshold of $150 \%$ CD86 induction, which is the basis for the EC150 calculation, is indicated as a solid line. Cell viability was above $50 \%$ of the negative control, except for the concentrations labeled with an asterisk $\left({ }^{*}\right)$.
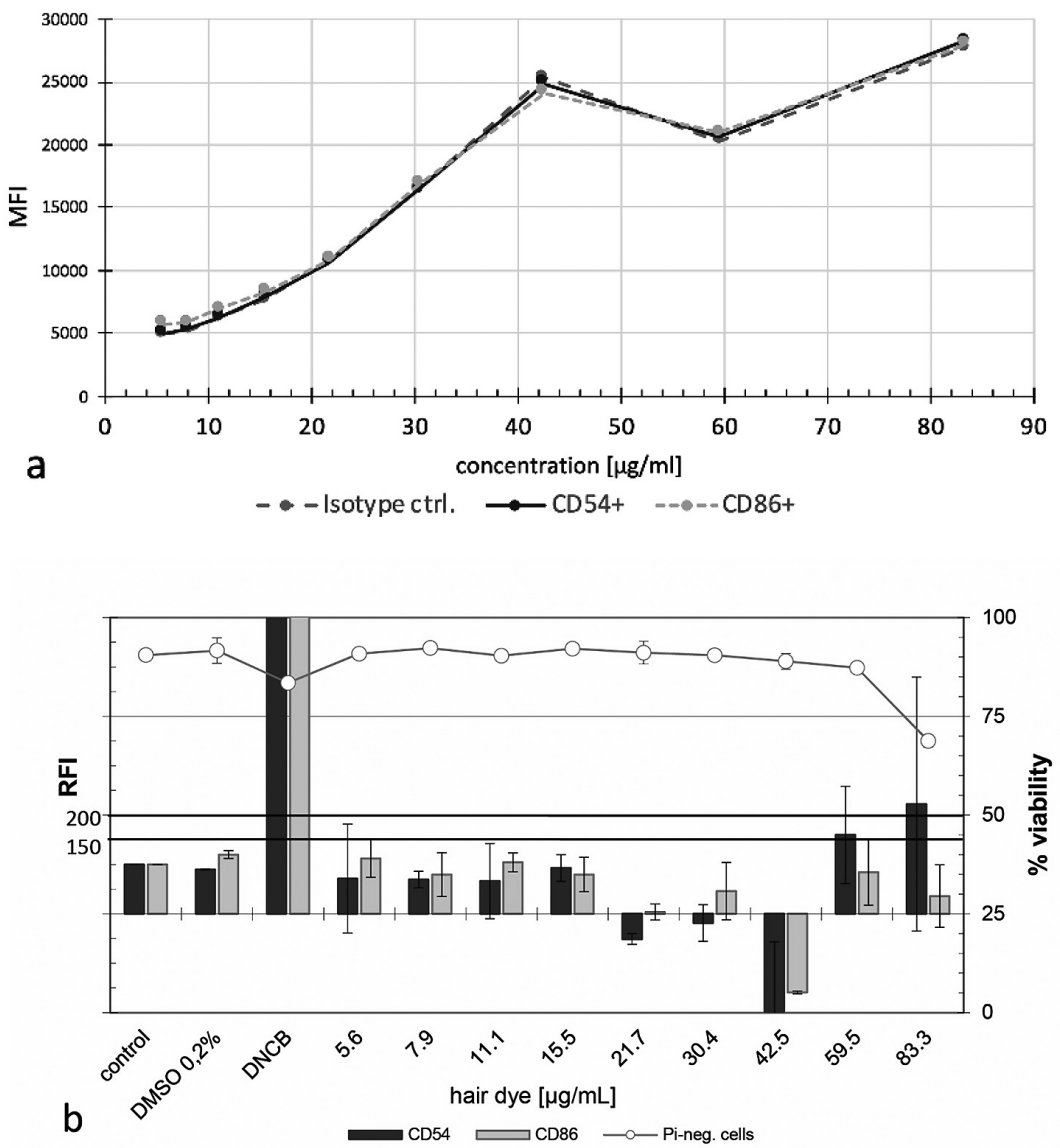

Fig. 8: Typical dose-response curves for THP-1 cells exposed to the proprietary oxidative hair dye and labeled with FITCtagged antibodies against CD54 or CD86 or with the isotype control antibody

(a) Mean fluorescence intensity (MFI) values for 9 concentrations of the hair dye, determined in channel FL1. (b) Corresponding relative fluorescence intensity (RFI; bars) and relative cell viability (\% of negative control; solid line with open circles) values for negative, positive, and solvent control, and for 8 concentrations of the hair dye. The solid black lines indicate the $150 \%$ and $200 \%$ induction thresholds, respectively. 

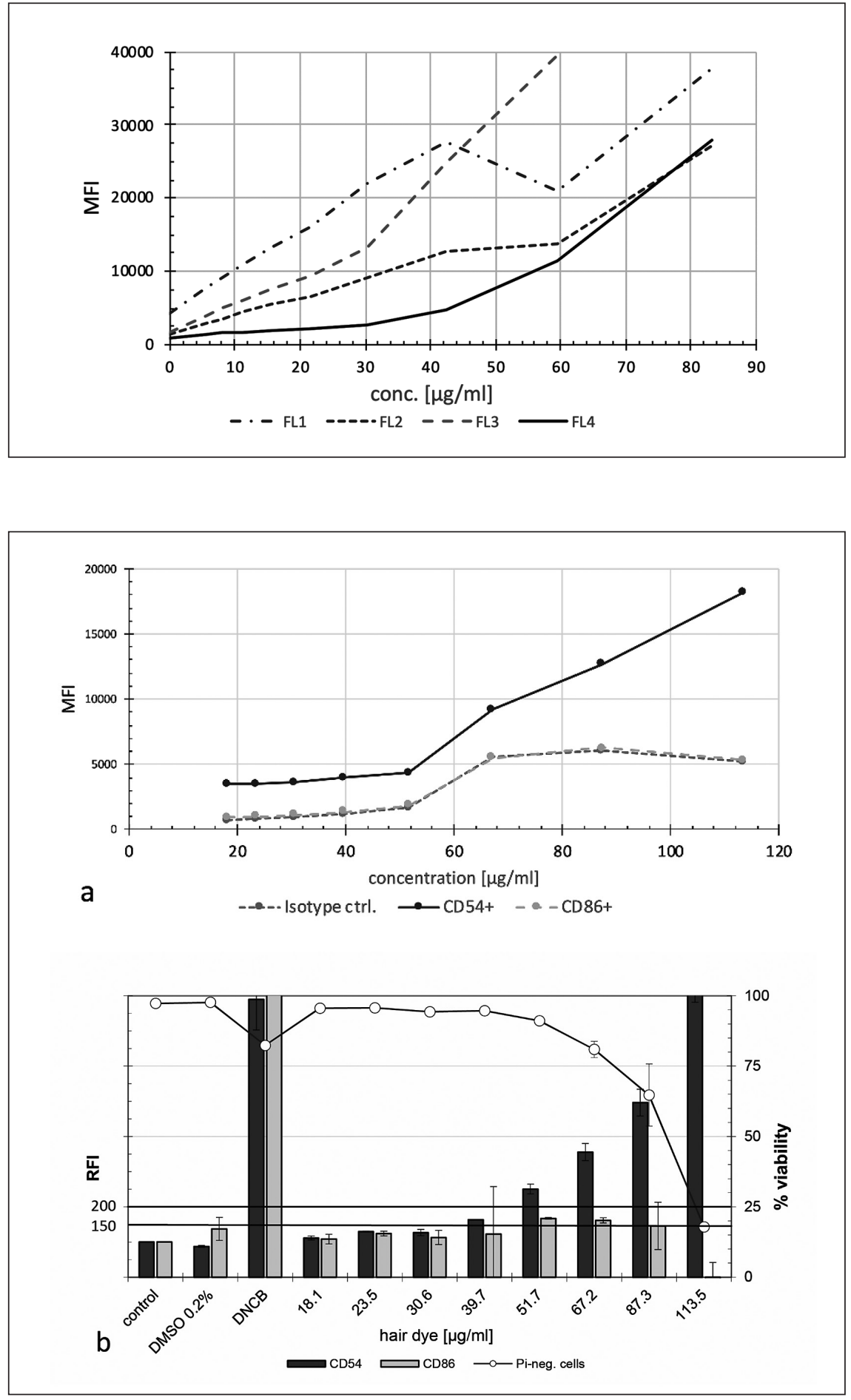

Fig. 9: Dose-response curves of THP-1 cells after exposure to the oxidative hair dye (fluorescence check) in the absence of antibodies

The mean fluorescence intensity (MFI) was determined in all 4 flow cytometer channels (FL1-FL4).

Fig. 10: Typical dose-response curves for THP-1 cells exposed to the proprietary oxidative hair dye and labeled with APC-tagged antibodies against CD54 or CD86 or with the isotype control antibody (a) Mean fluorescence intensity (MFI) values for 8 concentrations of the hair dye, determined in flow cytometer channel FL4. (b) Corresponding relative fluorescence intensity (RFI; bars) and relative cell viability (\% of negative control; solid line with open circles) values for negative, positive and solvent control, and for 8 different hair dye concentrations. The solid black lines indicate the $150 \%$ and $200 \%$ induction thresholds, respectively. ture, with emission wavelengths similar to those of FITC when excited with the corresponding flow cytometer laser.

We speculated that APC-labeled antibodies against CD54 and CD86, with a fluorescence spectrum shifted to longer wavelengths compared to FITC, would circumvent the observed fluorescence interference. Therefore, as a proof-of-concept, we selected a presumably FITC-interfering oxidative hair dye (propri- etary chemical of Henkel AG \& Co. KGaA) and conducted the h-CLAT assay with antibodies labeled with either FITC or APC.

With FITC-labeled antibodies, the MFI of the isotype controls increased proportionally with increasing chemical concentrations. The MFI for CD54 and CD86 increased in a similar manner, thus the 3 curves illustrating the concentration-dependent increases in fluorescence intensity were nearly indistinguishable 
(Fig. 8a). Consequently, the RFI values were not much higher than the solvent control (defined as $100 \%$ RFI) or even negative values (Fig. 8b). Only for the highest chemical concentration tested, the RFI for CD54 crossed the $200 \%$ induction threshold once, although the individual test values differed markedly, illustrated by the large error bars. Hence, the test results must be considered inconclusive and no unambiguous statement about the test substance's skin sensitizing potential could be made.

For most chemicals tested so far, including the proficiency chemicals, the isotype control MFIs did not increase concentration-dependently as seen with the oxidative hair dye tested in this approach. This observation supports the autofluorescence hypothesis for the hair dye chemical. To investigate this further, we incubated THP-1 cells with the hair dye at 8 different concentrations, based on a previously conducted dose-range finder, for 24 hours and subsequently analyzed the fluorescence emission in all 4 available flow cytometer channels. Even at low concentrations, the MFI strongly increased in the FITC-related channel FL1 after blue laser excitation (Fig. 9). In contrast, lowest MFI values were observed in channel FL4, which detects fluorescence emission at wavelengths around $675 \mathrm{~nm}$ after red laser excitation, which is typical for fluorochromes like APC. Based on this "fluorescence check", we chose APC and the FL4 channel as the new configuration to generate more reliable h-CLAT data for the oxidative hair dye compared to the FITC approach.

Analyzing the CD54 and CD86 expression with APC-labeled antibodies led to results that strongly differed from those gained with FITC. The MFI of the isotype control did not change markedly at the lower concentrations tested, and a moderate increase appeared only at the 3 highest concentrations. The MFI values for CD86 were in a similar range as the isotype control, the curves illustrating the concentration-dependent change in MFI were nearly indistinguishable (Fig. 10a). However, the dye elicited a concentration-dependent increase in CD54 expression with MFI values clearly above those of the isotype control. After RFI calculation we observed CD54 expression of more than $200 \%$, compared to the medium control, for the 4 highest concentrations tested, with 3 concentrations resulting in cell viabilities above $50 \%$ (Fig. 10b). For CD86, the RFI of 2 concentrations slightly crossed the respective threshold of $150 \%$ compared to the medium control. Thus, according to the h-CLAT prediction model, all criteria were fulfilled to consider the oxidative hair dye a sensitizer.

\section{Discussion}

With this proficiency exercise we have demonstrated the similarity of FITC- and APC-labeled anti-CD54 and anti-CD86 antibodies with respect to h-CLAT performance and predictivity.

The h-CLAT is a key method in several defined approaches recently submitted to the OECD (OECD, 2019; Kleinstreuer et al., 2018; Hoffmann et al., 2018). Its use demands clear definitions of its applicability domain as well as of its limitations and pitfalls, otherwise reliability and credibility of the defined approach as such might be jeopardized in cases of challenging chemicals. Considering these demands, every study that adds information about h-CLAT performance for a certain type of chemical or that sug- gests reasonable and scientifically valid optimization steps, should be thoroughly assessed by the authorities. If it passes the review process successfully, it should become integrated in the existing test guideline and standard operation procedure, respectively.

Previous studies intended to broaden the applicability domain of or to introduce technological innovations into an already existing OECD-approved in vitro alternative method have already been published and subsequently submitted to the respective OECD expert groups (e.g., Emter and Natsch, 2015; Verstraelen et al., 2017; Edwards et al., 2018). Thus, with our current proficiency exercise for the h-CLAT, we continue this tradition of continuous improvement of alternatives to animal testing for an even better and more reliable safety assessment of chemicals.

Currently, the discussion about replacing animal-derived products with completely animal-free, recombinant components in in vitro methods is gaining momentum. New animal-free products are entering the market and can now be validated against the historical ones. However, in terms of an OECD-approved test method like the h-CLAT, changing one essential component of the protocol must be justified and supported by data generated in a comparative proficiency exercise. In this study we focused exclusively on the exchange of the fluorochromes coupled to the antibodies, an integral part of the test method, while otherwise strictly following the h-CLAT protocol. This included the use of fetal bovine serum and antibodies derived from mammalian cell culture, both mandatory components of the DB-ALM protocol No. 158 in the version of 2014, which was the valid protocol when we started the study. If more than one parameter had been changed simultaneously, the cause-effect principle would have been compromised and, in case of doubt, no reliable conclusion about the fluorochrome performance could have been drawn from our analysis. The measures to adapt the h-CLAT to animal-free products were published only after our comparative study had been finalized (Edwards et al., 2018).

The h-CLAT prediction model defines chemicals as non-sensitizers when the relative fluorescence intensity of all dilutions tested remains below the thresholds for CD54 and CD86 induction and cell viability is higher than 50\%. However, several studies with larger sets of chemicals have revealed certain false-negative predictions when compared with either the murine local lymph node assay or human data (Basketter et al., 2014; Hoffmann et al., 2018; Kleinstreuer et al., 2018). In the process of risk assessment, it is of utmost importance to distinguish between true and false negatives. Thus, OECD TG 442E defines the applicability domain to exclude chemicals that are prone to false-negative predictions.

On the one hand, these are lipophilic substances with a $\log P$ $>3.5$. When analyzing the predictive parameters for 143 chemicals, including 31 substances with a $\log P$ value $>3.5$, sensitivity and accuracy markedly increased when the 31 lipophilic chemicals were not considered in the calculation (Takenouchi et al., 2013). Nine chemicals with $\log P$ values $>3.5$ were classified false-negative in the h-CLAT. In contrast, biolipids, namely unsaturated and saturated fatty acids, tend to be overpredicted in the h-CLAT, but also in other skin sensitization assays such as the Keratinosens ${ }^{\mathrm{TM}}$ and the DPRA test, when compared with in vivo data generated in the guinea pig maximization test (Kreiling et al., 2017). 
On the other hand, chemicals emitting fluorescence in the same wavelength ranges as FITC or propidium iodide tend to fall out of the applicability domain. For example, this affects some oxidative hair dyes like $\mathrm{p}$-phenylenediamine derivatives, including the proprietary hair dye tested in this study, which, due to their chemical structure, are highly reactive and tend to form diand trimers with quinone structures (personal communication). The ability of the h-CLAT to correctly predict the sensitizing potential of hair dyes was investigated by Okamoto and colleagues (2010), who had developed the original h-CLAT protocol. They tested nine different hair dyes, including p-phenylenediamine and Bandrowski's base, a known strong sensitizer. The test runs were performed with FITC-labeled antibodies and PI. All chemicals were predicted correctly as sensitizers. Although a concentration-dependent increase in the RFI of the isotype control was observed, RFI values above the respective thresholds for CD54 and CD86 at some concentrations were unambiguous indicators for sensitizers. The authors concluded that oxidative hair dyes fall into the applicability domain of the h-CLAT assay since no false-negative results were obtained even with autofluorescent test chemicals.

These results stood in clear contrast to our observations for the proprietary hair dye. The concentration-dependent increase of MFI values in the isotype control was a clear indication for autofluorescence, which, according to Okamoto et al. (2010), could be tested in the h-CLAT. However, when, after subtracting the isotype controls from the MFI values of CD54 and C86, RFI values were below the thresholds or even negative, no conclusion about the sensitizing potential of the test item could be drawn. Fortunately, according to OECD TG 442E (OECD, 2018), alternative fluorochromes with different spectral properties can be applied in the h-CLAT assay if their equivalence with FITC and/or propidium iodide has been demonstrated in a proficiency exercise.

With the proficiency exercise, which is the subject of this paper, we have proven that FITC-labeled anti-CD54 and anti-CD86 antibodies can be successfully replaced with APC-labeled antibodies without any loss in predictivity and potency estimation (EC150 and EC200 values). The high degree of similarity of the MFI values for FITC and APC, a fluorochrome with an emission spectrum at longer wavelengths, was emphasized by high correlation coefficients for both sensitizers and non-sensitizers but also for the isotype controls. Comparing the MFI values generated with identical cells under identical exposure conditions allowed the characterization of the specific light-emitting properties of the antibodies labeled with either FITC or APC. Because of the otherwise identical experimental conditions, any differences in MFI depended only on the emission properties of the two fluorochromes. Light emission after laser excitation was generally more intense with the FITC fluorochrome, resulting in higher MFI values compared to APC. However, the observed difference in the physical properties of the investigated fluorochromes did not impact the predictivity of the h-CLAT assay. The classification of a chemical as a sensitizer or non-sensitizer is based on the RFI, which is the respective MFI value standardized against the isotype control. Because the differences in the light emission properties affected the isotype control MFIs in the same way as the CD54/86 measurements, the RFI values for both fluorochromes were in comparable orders of magnitude and correlated well with respect to the sensitizers.

In contrast, the RFI values for the non-sensitizers appeared to be poorly correlated. However, this result is not unexpected because non-sensitizers do not induce CD54 and CD86 above the defined thresholds of $150 \%$ and $200 \%$, respectively. Thus, the RFI data acquire random values comparable to the negative or solvent controls without any apparent dose dependency. This is reflected by the low correlation coefficient.

As a consequence of the similar performance of FITC- and APC-labeled antibodies, all chemicals were classified correctly according to the known sensitizing potential. It became evident that the few negative differences between the MFI values for FITC and APC, calculated for CD54 (see Fig. 3), were related to very high absolute MFI values for both FITC and APC, which unambiguously classified the tested chemicals as sensitizers. At this high level of CD54 induction, the MFI difference, and hence the light emission properties of the fluorochromes, were not relevant for the final classification.

To further analyze the apparent differences in the light emission characteristics between FITC and APC, we conducted additional flow cytometry tests with naïve THP-1 cells, which were either labeled with the different antibodies or unstained. In general, the MFI values for FITC were always higher than those for APC. Even the untreated and unlabeled THP-1 cells revealed a certain degree of autofluorescence, with higher MFI values in the FL1 channel (FITC) compared to the FL4 channel (APC). Thus, the background for the FITC measurements is higher than for the APC analysis, based on both the specific physical-chemical properties of the fluorochromes and, to a certain degree, on the autofluorescence of the THP-1 cells. In addition, the FITC- and APC-labeled anti-CD86 antibodies were derived from the same clone and thus have identical antigen binding sites. This might be the reason for the more homogeneous results with exclusively positive MFI differences for CD86, whereas for CD54, with antibodies from different clones, also negative differences were found. The higher variability of FITC versus APC differences observed for sensitizers might reflect the natural biological variation limits of the individual THP-1 cells in terms of CD54 and CD86 expression when exposed to this kind of chemicals.

Interestingly, upon exposure to 2 out of the 10 proficiency chemicals only one of the two CD proteins was expressed at increased levels. The strong sensitizer 1,4-PD induced only CD86, but not CD54. This is in accordance with the reference ranges for EC150 and EC200 provided in OECD TG 442E. It states that for this chemical "a majority of negative results have been obtained for this marker and a negative result is mostly expected" (OECD, 2018). Upon exposure to $\mathrm{R}(+)$-limonene, a weak sensitizer, only CD54 was expressed, whereas no induction was observed for CD86, which also matches the OECD TG 442E reference ranges based on historical data. However, the EC200 values for both FITC- and APC-labeled antibodies exceeded the recommended reference range nearly twofold, which possibly could be attributed to solubility issues of $\mathrm{R}(+)$-limonene. At higher concentrations, this chemical tended to form emulsions instead of clear 
solutions in the cell culture medium, which could affect its bioavailability in the h-CLAT assay. Based on this consideration, it can be expected that higher substance concentrations are needed to induce CD54 above the respective threshold.

Among the proficiency chemicals listed in OECD TG 442E, 2-MBT, a moderate sensitizer, is the third substance with documented differences in CD54 and CD86 expression. However, in contrast to the information on a majority of negative historical test results provided in the guideline, CD86 expression above the $150 \%$ threshold was observed with the APC-labeled antibodies in the current study, resulting in an EC150 value in the same order of magnitude as the corresponding EC200 values. With the FITC antibodies, no significant CD86 increase occurred and hence no EC150 could be calculated. It became evident that a clear dose dependency of CD86 expression was missing. While some of the RFI values generated with the FITC antibodies in the 2 test runs nearly reached but never crossed the $150 \%$ threshold, a few APCbased values exceeded the $150 \%$ expression level, leading to an EC150 value. Under these circumstances, even small variations in the RFI values can have a huge impact on the EC150, depending on which RFI values at which substance concentrations cross the respective threshold or not. With RFI data close to the threshold, this could easily be considered a matter of intrinsic variability of biological systems. Thus, the difference in CD86 expression between FITC and APC does not necessarily reflect differences in the antibody performance, but more likely the uncertainty in the EC150 calculation based on the specific dose-response curve for 2-MBT.

The same reason can be applied to explain the difference in CD86 expression between both fluorochromes observed with 1,4-PD. Lacking clear dose dependency and RFI values in the near vicinity of the $150 \%$ threshold introduced a degree of uncertainty in the EC150 calculation, which is mirrored in larger EC150 concentration differences than would be expected. However, even the high APC-based value is located well within the reference ranges indicated by OECD TG 442E. Taken together, the apparent differences between the EC150/200 values are less the result of differences in the antibody/fluorochrome performance, but originate more in the specific protein expression patterns of the THP-1 cells when exposed to the respective sensitizing chemicals under the conditions described in this paper.

The situation that only one of the characteristic marker molecules is expressed after exposure sometimes occurs with other chemicals too (own observations), suggesting a special physiological mechanism of THP-1 cell activation. It is also reflected in the respective h-CLAT prediction model, which states that either CD54 or CD86 or both must be overexpressed to indicate a sensitizer. However, what is important in this respect is the observation that the same results in terms of classification were achieved with the FITC- and the APC-labeled antibodies, again proof for the equivalence of the antibodies employed.

By demonstrating the equivalence of APC- and FITC-labeled antibodies using the proficiency chemicals, the key demand of OECD TG 442E for the use of alternative fluorochromes was thus fulfilled. The positive outcome of our study has certainly benefitted from the convenient situation that the antibodies were from two companies only, with APC- and FITC-labeled CD86 antibodies from the same provider and the same clone. Working with alternative antibodies from other sources might lead to deviating results. If there is doubt whether h-CLAT results generated with other antibodies/fluorochromes are reliable, a proficiency exercise similar to the one we have run should be mandatory.

The advantage of using APC instead of FITC was demonstrated in the analysis of an autofluorescent oxidative hair dye of previously unknown in vivo sensitization potential and potency (proprietary to Henkel). After having obtained inconclusive results based on the FITC-tagged antibodies, we conducted a fluorescence check to determine which of the detection channels available in the flow cytometer was affected least by the autofluorescence. As this was the case for a filter with highest sensitivity in the red wavelengths range, we selected APC as the fluorochrome of choice with corresponding spectral properties.

With APC, the fluorescence signals of CD54 expression in the THP-1 cells were clearly distinguishable against the isotype fluorescence background. Consequently, the hair dye must be considered a potential sensitizer, following the h-CLAT prediction model. CD86, in contrast, seemed not to be significantly induced by the hair dye, its dose-response curve was nearly identical to that of the isotype control.

Before running the h-CLAT for assessing the skin-sensitizing potential of substances that are suspected to have autofluorescent properties, we therefore recommend adding a fluorescence check as a mandatory working step at the beginning of the h-CLAT protocol. Briefly, THP-1 cells should be exposed to the chemical at 8 concentrations for 24 hours and then be analyzed in all available laser/channel combinations. Then the combination with the lowest signals should be selected and a matching fluorochrome chosen. The details are given in Section 2. With this additional working step, the applicability domain of the h-CLAT as depicted in the OECD TG 442E can be extended to a family of chemicals which, owing to their physical-chemical properties, were not compatible with this in vitro alternative method previously.

\section{Conclusion}

Taken together, replacing FITC-labeled antibodies with APCtagged antibodies in the h-CLAT assay resulted in identical classifications of the proficiency chemicals as sensitizers or non-sensitizers. With this proficiency exercise, a prerequisite of the TG that demands to prove the equivalence in performance of alternative antibodies and fluorochromes compared to the standard FITC-labeled antibodies before running safety assessment studies is fulfilled. In addition, by having demonstrated the equivalence of FITC- and APC-labeled antibodies, the applicability domain of the h-CLAT assay could be significantly extended for chemicals which, due to autofluorescence in the FITC spectral emission range, were previously incompatible with this OECD-approved in vitro skin sensitization assay. These findings pave the way to a more realistic and reliable in vitro safety assessment for challenging autofluorescent chemicals using the h-CLAT assay. 


\section{References}

Basketter, D. A., Alépée, N., Ashikaga, T. et al. (2014). Categorization of chemicals according to their relative human skin sensitizing potency. Dermatitis 25, 11-21. doi:10.1097/DER. 0000000000000003

DB-ALM (2014). Protocol No. 158: Human Cell Line Activation Test (h-CLAT). https://bit.ly/35ThGVS

Diepgen, T. L., Ofenloch, R. F. and Bruze, M. (2016). Prevalence of contact allergy in the general population in different European regions. Br J Dermatol 174, 319-329. doi:10.1111/bjd.14167

EC EURL ECVAM (2012). Human Cell Line Activation Test (h-CLAT) Validation Study Report. https://eurl-ecvam.jrc.ec. europa.eu/eurl-ecvam-recommendations

Edwards, A., Roscoe, L., Longmore, C. et al. (2018). Adaptation of the human cell line activation test (h-CLAT) to animal-product-free conditions. ALTEX 35, 477-488. doi:10.14573/altex. 1710051

Emter, R. and Natsch, A. (2015). A fast resazurin-based live viability assay is equivalent to the MTT-test in the KeratinoSens assay. Toxicol In Vitro 29, 688-693. doi:10.1016/j.tiv. 2015.02.003

EU - European Union (2009). Regulation (EC) No 1223/2009 of the European Parliament and of the Council of 30 November 2009 on cosmetic products (2019). https:/eur-lex. europa.eu/legal-content/EN/TXT/PDF/?uri=CELEX: 02009R1223-20191127\&from $=\mathrm{EN}$

Hoffmann, S., Kleinstreuer, N., Alépée, N. et al. (2018). Non-animal methods to predict skin sensitization (I): The Cosmetics Europe database. Crit Rev Toxicol 48, 344-358. doi:10.1080/1 0408444.2018.1429385

Kleinstreuer, N. C., Hoffmann, S., Alépée, N. et al. (2018). Non-animal methods to predict skin sensitization (II): An assessment of defined approaches. Crit Rev Toxicol 48, 359-374. doi:10.1080/10408444.2018.1429386

Kosaka, N., Okamoto, K., Miziuno, M. et al. (2008). A study of the criteria for selection of THP-1 cells in the human cell line activation test (h-CLAT): Results of the $2^{\text {nd }}$ Japanese interlaboratory study. AATEX 13, 33-62. http://www.asas.or.jp/ jsaae_old/aatex/13-2-2.pdf

Kreiling, R., Gehrke, H., Broschard, T. H. et al. (2017). In chemico, in vitro and in vivo comparison of the skin sensitizing potential of eight unsaturated and one saturated lipid compounds. Regul Toxicol Pharmacol 90, 262-276. doi:10.1016/j. yrtph.2017.09.023

Langen, U., Schmitz, R. and Steppuhn, H. (2013). Prevalence of allergic diseases in Germany: Results of the German health interview and examination survey for adults (DEGS1). Bundesgesundheitsblatt Gesundheitsforschung Gesundheitsschutz 56,
698-706 [Article in German]. doi:10.1007/s00103-012-1652-7

Nukada, Y., Ashikaga, T., Miyazawa, M. et al. (2012). Prediction of skin sensitization potency of chemicals by human cell line activation test (h-CLAT) and an attempt at classifying skin sensitization potency. Toxicol In Vitro 26, 1150-1160. doi:10.1016/j.tiv.2012.07.001

OECD (2014). The Adverse Outcome Pathway for Skin Sensitisation Initiated by Covalent Binding to Proteins. OECD Series on Testing and Assessment, No. 168. OECD Publishing, Paris. doi:10.1787/9789264221444-en

OECD (2018). Test No. 442E: In Vitro Skin Sensitisation: In Vitro Skin Sensitisation assays addressing the Key Event on activation of dendritic cells on the Adverse Outcome Pathway for Skin Sensitisation. OECD Guidelines for the Testing of Chemicals, Section 4. OECD Publishing, Paris. doi:10. 1787/9789264264359-en

OECD (2019). Draft OECD guideline defined approaches for skin sensitisation. https://www.oecd.org/env/ehs/testing/ GL\%20DASS_22Sep2019v2.pdf

Okamoto, K., Kato, Y., Kosaka, N. et al. (2010). The Japanese ring study of a human cell line activation test (h-CLAT) for predicting skin sensitization potential ( $6^{\text {th }}$ report): A study for evaluation oxidative hair dye sensitization potential using the h-CLAT. AATEX 15, 81-88. https://www.jstage.jst.go.jp/article/ aatex/15/2/15_2_81/_pdf

Peiser, M., Tralau, T., Heidler, J. et al. (2012). Allergic contact dermatitis: Epidemiology, molecular mechanisms, in vitro methods and regulatory aspects. Current knowledge assembled at an international workshop at BfR, Germany. Cell Mol Life Sci 69, 763-781. doi:10.1007/s00018-011-0846-8

Sakaguchi, H., Ryan, C., Ovigne, J. M. et al. (2010). Predicting skin sensitization potential and inter-laboratory reproducibility of a human cell line activation test (h-CLAT) in the European cosmetics association (COLIPA) ring trials. Toxicol In Vitro 24, 1810-1820. doi:10.1016/j.tiv.2010.05.012

Takenouchi, O., Miyazawa, M., Saito, K. et al. (2013). Predictive performance of the human cell line activation test (h-CLAT) for lipophilic chemicals with high octanol-water partition coefficients. J Toxicol Sci 38, 599-609. doi:10.2131/jts.38.599

Verstraelen, S., Maglennon, G., Hollanders, K. et al. (2017). CON4EI: Bovine corneal opacity and permeability (BCOP) test for hazard identification and labelling of eye irritating chemicals. Toxicol In Vitro 44, 122-133. doi:10.1016/j. tiv.2017.06.028

\section{Conflicts of interest}

The authors declare that they have no conflicts of interest. 